\title{
A global attractor for a fluid-plate interaction model accounting only for longitudinal deformations of the plate
}

\author{
Igor Chueshov* \\ Department of Mechanics and Mathematics, \\ Kharkov National University, \\ Kharkov, 61077, Ukraine
}

September 21, 2021

\begin{abstract}
We study asymptotic dynamics of a coupled system consisting of linearized 3D Navier-Stokes equations in a bounded domain and the classical (nonlinear) elastic plate equation for in-plane motions on a flexible flat part of the boundary. The main peculiarity of the model is the assumption that the transversal displacements of the plate are negligible relative to in-plane displacements. This kind of models arises in the study of blood flows in large arteries. Our main result states the existence of a compact global attractor of finite dimension. We also show that the corresponding linearized system generates exponentially stable $C_{0}$-semigroup. We do not assume any kind of mechanical damping in the plate component. Thus our results means that dissipation of the energy in the fluid due to viscosity is sufficient to stabilize the system.

Keywords: Fluid-structure interaction, linearized 3D Navier-Stokes equations, nonlinear plate, finite-dimensional attractor.
\end{abstract}

2010 MSC: 35Q30, 74F10

\section{Introduction}

We consider a coupled (hybrid) system which describes interaction a homogeneous viscous incompressible fluid which occupies a domain $\mathcal{O}$ bounded by the (solid) walls of the container $S$ and a horizontal boundary $\Omega$ on which a thin (nonlinear) elastic plate is placed. The motion of the fluid is described by linearized 3D Navier-Stokes equations. To describe deformations of the plate we involve a general (full) Kirchhoff-Karman type model (see, e.g.,

*e-mail: chueshov@univer.kharkov.ua 
[25, 26, 27] and the references therein) with additional hypotheses that the transversal displacements of the plate are negligible relative to in-plane displacements. Thus we only consider longitudinal deformations of the plate and take account of tangential shear forces which fluid exerts on the plate. This kind of models arises in the study of the problem of blood flows in large arteries (see, e.g., 30] and also [17] for a more recent discussion and references).

We note that the mathematical studies of the problem of fluid-structure interaction in the case of viscous fluids and elastic plate/bodies have a long history. We refer to [8, 17, 18, 19, 23] and the references therein for the case of plates/membranes, to [15] in the case of moving elastic bodies, and to [1, 2, 3, 5, 6, 16] in the case of elastic bodies with the fixed interface; see also the literature cited in these references.

Our mathematical model is formulated as follows.

Let $\mathcal{O} \subset \mathbb{R}^{3}$ be a bounded domain with a sufficiently smooth boundary $\partial \mathcal{O}$. We assume that $\partial \mathcal{O}=\Omega \cup S$, where $\Omega \subset\left\{x=\left(x_{1} ; x_{2} ; 0\right):\left(x_{1} ; x_{2}\right) \in\right.$ $\left.\mathbb{R}^{2}\right\}$ and $S$ is a smooth surface which lies under plane $x_{3}=0$. The exterior normal on $\partial \mathcal{O}$ is denoted by $n$. We have that $n=(0 ; 0 ; 1)$ on $\Omega$. We consider the following linear Navier-Stokes equations in $\mathcal{O}$ for the fluid velocity field $v=v(x, t)=\left(v^{1}(x, t) ; v^{2}(x, t) ; v^{3}(x, t)\right)$ and for the pressure $p(x, t)$ :

$$
\begin{gathered}
\partial_{t} v-\nu \Delta v+\nabla p=\tilde{G} \quad \text { in } \mathcal{O} \times(0,+\infty), \\
\operatorname{div} v=0 \quad \text { in } \mathcal{O} \times(0,+\infty), \\
v(x, 0)=v_{0}(x) \quad \text { in } \mathcal{O},
\end{gathered}
$$

where $\nu>0$ is the dynamical viscosity and $\tilde{G}$ is a volume force. We supplement (11)-(3) with the (non-slip) boundary conditions imposed on the velocity field $v=v(x, t)$ :

$$
v=0 \quad \text { on } S ; \quad v \equiv\left(v^{1} ; v^{2} ; v^{3}\right)=\left(u_{t} ; 0\right) \equiv\left(u_{t}^{1} ; u_{t}^{2} ; 0\right) \text { on } \Omega,
$$

where $u=u(x, t) \equiv\left(u^{1}(x, t) ; u^{2}(x, t)\right)$ is the in-plane displacement vector of the plate placed on $\Omega$ satisfying the following equations (see, e.g., [26] and [27]):

$$
\rho h u_{t t}^{1}-\left(\partial_{x_{1}} N_{11}+\partial_{x_{2}} N_{12}\right)+F^{1}=0 \text { in } \Omega \times(0, \infty),
$$

and

$$
\rho h u_{t t}^{2}-\left(\partial_{x_{1}} N_{21}+\partial_{x_{2}} N_{22}\right)+F^{2}=0 \text { in } \Omega \times(0, \infty),
$$

with

$$
N_{11}=D\left(u_{x_{1}}^{1}+\mu u_{x_{2}}^{2}\right), \quad N_{22}=D\left(u_{x_{2}}^{2}+\mu u_{x_{1}}^{1}\right)
$$

and

$$
N_{12}=N_{21}=\frac{D}{2}(1-\mu)\left(u_{x_{2}}^{1}+u_{x_{1}}^{2}\right),
$$


where $D=E h /\left(1-\mu^{2}\right), E$ is Young's modulus, $0<\mu<1 / 2$ is Poisson's ratio, $h$ is the thickness of the plate, $\rho$ is the mass density. The external (in-plane) force $\left(F^{1} ; F^{2}\right)$ in (5) and (6) consists of two parts,

$$
F^{i}=f^{i}\left(u^{1}, u^{2}\right)+T_{i}(v), \quad i=1,2,
$$

where $\left(f^{1}\left(u^{1}, u^{2}\right) ; f^{2}\left(u^{1}, u^{2}\right)\right)$ is a nonlinear feedback force represented by some potential $\Phi$ (which we specify below):

$$
f^{i}\left(u^{1}, u^{2}\right)=\frac{\partial \Phi\left(u^{1}, u^{2}\right)}{\partial u^{i}}, \quad i=1,2,
$$

and $\left(T_{1}(v) ; T_{2}(v)\right)$ is the viscous shear stress exerted by the fluid on the plate, $T_{i}(v)=\left(\left.(T n)\right|_{\Omega}, e_{i}\right)_{\mathbb{R}^{3}}$. Here $T=\left\{T_{i j}\right\}_{i, j=1}^{3}$ is the stress tensor of the fluid,

$$
T_{i j} \equiv T_{i j}(v)=\nu\left(v_{x_{j}}^{i}+v_{x_{i}}^{j}\right)-p \delta_{i j}, \quad i, j=1,2,3,
$$

$e_{1}=(1 ; 0 ; 0), e_{2}=(0 ; 1 ; 0)$ are unit tangential vectors on $\Omega \subset \partial \mathcal{O}$ and $n=(0 ; 0 ; 1)$ is the outer normal vector to $\partial \mathcal{O}$ on $\Omega$. A simple calculation shows that

$$
T_{i}(v) \equiv T_{i 3}(v)=\nu\left(v_{x_{3}}^{i}+v_{x_{i}}^{3}\right)=\nu v_{x_{3}}^{i}, \quad i=1,2,
$$

(in the last equality we use the fact that $v^{3}\left(x_{1} ; x_{2} ; 0\right)=0$ for $\left(x_{1} ; x_{2}\right) \in \Omega$ due to the second relation in (4) and hence $v_{x_{i}}^{3}=0$ on $\left.\Omega, i=1,2\right)$.

Thus we arrive at the following equations for the in-plane displacement $u=\left(u^{1} ; u^{2}\right)$ of the plate (below for some notational simplifications we assume that $\rho h=1$ and $D(1-\mu) / 2=1)$ :

$$
u_{t t}^{i}-\Delta u^{i}-\lambda \partial_{x_{i}}[\operatorname{div} u]+\left.\nu v_{x_{3}}^{i}\right|_{x_{3}=0}+f^{i}(u)=0, \quad i=1,2,
$$

where $\lambda=(1+\mu)(1-\mu)^{-1}$ is a nonnegative parameter. For the displacement $u=\left(u^{1} ; u^{2}\right)$ we impose the clamped boundary conditions on $\Gamma=\partial \Omega$ :

$$
u^{i}=0 \text { on } \Gamma, \quad i=1,2 .
$$

Our main point of interest is wellposedness and long-time dynamics of solutions to the coupled problem in (1)-(4), (7), and (8) for the velocity $v$ and the displacement $u=\left(u^{1} ; u^{2}\right)$ with the initial data

$$
\left.v\right|_{t=0}=v_{0},\left.\quad u\right|_{t=0}=u_{0},\left.\quad u_{t}\right|_{t=0}=u_{1} .
$$

This problem in the case when $\tilde{G} \equiv 0, \lambda=0$ and $f^{i}(u) \equiv 0$ was considered in [19] (see also [17, 18]) with an additional strong (Kelvin-Voight type) damping force applied to the interior of the plate. These papers deals with the existence and asymptotic stability of the corresponding semigroup. In contrast with [17, 18, 19] we do not assume the presence of mechanical damping 
terms in the plate component of the system and consider nonlinearly forced model.

Our main result (see Theorem 4.1) states that under some natural conditions concerning the potential $\Phi(u)$ of feedback forces system (11)-(4) and (77) - (9) possesses a compact global attractor of finite fractal dimension which also has some additional regularity properties. Our considerations involves recently developed approach based on quasi-stability properties and stabilizability estimates (see, e.g., [10, 12, 14] for the second order in time evolution equations and also [7, 13] for thermoelastic problems).

We also improve substantially the stability results presented in [17, 18, 19]. Namely, as a consequence of dissipativity property of the nonlinear model we prove (see Corollary 4.4) that linear part of (11)-(41) and (7)-(9) generates uniformly exponentially stable $C_{0}$-semigroup of contractions without any dissipation mechanisms (except the fluid viscosity) in the system.

We note that a linear model related to (1)-(4) and (7)-(9) was considered in [28, Section 3.15] from the point of view of boundary control. In [28] the authors deal with the so-called linearized model of well/reservour coupling with monophasic flow. This is a scalar linear model represented by the diffusion (heat) equation in $\mathcal{O}$ coupled with the wave equation on $\Omega$ with an interface condition like in (44). The scalar structure of the model makes it possible to prove [28, Proposition 3.15.5] that this model generates contractive exponentially stable analytic semigroup. We do not know whether the result on analyticity remains true for the hydrodynamical situation we consider. We also note that in fact the long time dynamics in problem (17) with a given stationary smooth velocity field $v$ was studied in [11] and [12, Chapter 7].

The paper is organized as follows. In the next Section 2 we discuss an auxiliary Stokes problem and rewrite problem (11)-(41) and (7)-(9) as a single first order equation in some extended space $\mathcal{H}$. Then we prove that the linear version of the problem generates strongly continuous contractive semigroup in $\mathcal{H}$. In Section 3 we prove the existence of strong and generalized solutions to the original nonlinear problem and establish energy balance identity. For this we use ideas presented in [29]. Section 4 contains our main results on long-time dynamics of system (11)-(4) and (7)-(9). Our considerations here are based mainly on the idea of quasi-stability (see, e.g., [12] and [14, Section 7.9]).

\section{Preliminaries}

In this section we first provide with some results concerning to Stokes problem and then consider the abstract form of the system. Below we denote by $H^{s}(D)$ the Sobolev space of the order $s$ on the set $D$, by $H_{0}^{s}(D)$ the closure of $C_{0}^{\infty}(D)$ in $H^{s}(D)$, and by $H^{s}(D) / \mathbb{R}$ the factor-space with the naturally 
induced norm.

\subsection{Stokes problem}

In further considerations we need some regularity properties of the terms responsible for fluid-plate interaction. For this we consider the following Stokes problem

$$
\begin{aligned}
& -\nu \Delta v+\nabla p=g, \quad \operatorname{div} v=0 \quad \text { in } \mathcal{O} \\
& v=0 \text { on } S ; \quad v=(\psi ; 0) \text { on } \Omega,
\end{aligned}
$$

where $g \in\left[L^{2}(\mathcal{O})\right]^{3}$ and $\psi=\left(\psi^{1} ; \psi^{2}\right) \in\left[L^{2}(\Omega)\right]^{2}$ are given. This type of boundary value problems for the Stokes equation was studied by many authors (see, e.g., 24] and [33] and the references therein). We collect some properties of solutions to (10) in the following assertion.

Proposition 2.1 Let $g \in\left[L^{2}(\mathcal{O})\right]^{3}$ and $\psi \in\left[H_{0}^{1}(\Omega)\right]^{2}$. Then

(1) Problem (10) has a unique solution $\{v ; p\} \in\left[H^{3 / 2}(\mathcal{O})\right]^{3} \times\left[H^{1 / 2}(\mathcal{O}) / \mathbb{R}\right]$ such that

$$
\|v\|_{\left[H^{1+s}(\mathcal{O})\right]^{3}}+\|p\|_{H^{s}(\mathcal{O}) / \mathbb{R}} \leq c_{0}\left\{\|g\|_{\left[H^{-1+s}(\mathcal{O})\right]^{3}}+\|\psi\|_{\left[H^{s+1 / 2}(\Omega)\right]^{2}}\right\}
$$

for every $0 \leq s \leq 1 / 2$. Moreover,

(2) We have that $\gamma_{\Omega} v \in\left[H^{-1 / 2}(\Omega)\right]^{2}$ for the trace operator $\gamma_{\Omega}$ defined (on smooth functions) by the formula

$$
\gamma_{\Omega} v=\nu\left(v_{x_{3}}^{1} ; v_{x_{3}}^{2}\right) \quad \text { for } \quad v=\left(v^{1} ; v^{2} ; v^{3}\right) \in\left[H^{2}(\mathcal{O})\right]^{3} .
$$

(3) If $g=0$ we also have that

$$
\|v\|_{\left[L_{2}(\mathcal{O})\right]^{3}} \leq c_{0}\|\psi\|_{\left[L_{2}(\Omega)\right]^{2}},
$$

thus we can define a bounded operator $N_{0}:\left[L_{2}(\Omega)\right]^{2} \mapsto\left[L^{2}(\mathcal{O})\right]^{3}$ by the formula

$$
N_{0} \psi=w \text { iff }\left\{\begin{array}{l}
-\nu \Delta w+\nabla p=0, \quad \operatorname{div} w=0 \quad \text { in } \quad \mathcal{O} \\
w=0 \text { on } S ; \quad w=(\psi ; 0) \text { on } \Omega
\end{array}\right.
$$

for $\psi=\left(\psi^{1} ; \psi^{2}\right) \in\left[L_{2}(\Omega)\right]^{2}\left(N_{0} \psi\right.$ solves (10) with $\left.g \equiv 0\right)$.

Proof. 1. The existence and uniqueness of solutions along with the bound in (11) follow from Proposition 2.3 and Remark 2.6 on Sobolev norm's interpolation in [33, Chapter 1].

2. To prove Statement 2 we use the same idea as in [1] which involves the boundary properties of harmonic functions (see [22]). 
We can represent $v$ in the form $v=\hat{v}+v^{*}$, where $\hat{v}$ solves (10) with $\psi \equiv 0$ and $v^{*}$ satisfies (10) with $g \equiv 0$. By Proposition 2.3 [33, Chapter 1]. we have that $\hat{v} \in\left[H^{2}(\mathcal{O})\right]^{3}$ and thus by the standard trace theorem there exists $\left.\partial_{n} \hat{v}\right|_{\partial \mathcal{O}} \in\left[H^{1 / 2}(\partial \mathcal{O})\right]^{3}$. Consequently $\gamma_{\Omega} \hat{v} \in\left[H^{1 / 2-\delta}(\Omega)\right]^{2} \subset\left[H^{-1 / 2}(\Omega)\right]^{2}$.

Thus we need to establish Statement 2 in the case $g \equiv 0$ only. In this case the pressure $p$ is a harmonic function in $\mathcal{O}$ which belongs $L_{2}(\Omega)$. Thus by Theorem 3.8.1 22, we can assign a meaning to $\left.p\right|_{\Omega}$ in $H^{-1 / 2}(\partial \mathcal{O})$. Now using the Gauss-Ostrogradskii formula one can see that $\nabla p \in\left(\left[H^{1}(\mathcal{O})\right]^{3}\right)^{\prime}$. Therefore from (10) with $g=0$ we have $\Delta v \in\left(\left[H^{1}(\mathcal{O})\right]^{3}\right)^{\prime}$. Using the Green formula

$$
\int_{\partial \mathcal{O}} \partial_{n} v^{i} \psi d S=\int_{\mathcal{O}} \Delta v^{i} \psi d x-\int_{\partial \mathcal{O}} \nabla v^{i} \nabla \psi d x, \quad \forall \psi \in H^{1}(\mathcal{O}),
$$

for every velocity component $v^{i}$, we conclude that $\gamma_{\Omega} v \in\left[H^{-1 / 2}(\Omega)\right]^{2}$.

3. To prove the third statement we use the representation of the velocity field with hydrodynamical potentials (see 24]). Indeed (see [24, Chapter 3, Section 2]), the velocity field $v=\left(v^{1} ; v^{2} ; v^{3}\right)$ solving (10) with $g \equiv 0$ admits the representation

$$
v^{i}(x)=\sum_{j=1}^{3} \int_{\partial \mathcal{O}} K_{i j}(x, y) \phi^{j}(y) d S_{y}, \quad i=1,2,3, x \in \mathcal{O},
$$

where $\phi=\left(\phi^{1} ; \phi^{2} ; \phi^{3}\right)$ satisfies the integral equation on $\partial \mathcal{O}$ :

$$
\frac{1}{2} \phi^{i}(x)+\sum_{j=1}^{3} \int_{\partial \mathcal{O}} K_{i j}(x, y) \phi^{j}(y) d S_{y}=\tilde{\psi}^{i}(x), \quad i=1,2,3, x \in \partial \mathcal{O} .
$$

Here $\tilde{\psi}$ is the extension of $(\psi ; 0)$ on $\partial \mathcal{O}$ by zero. The kernels $K_{i j}(x, y)$ has the form

$$
K_{i j}(x, y)=-\frac{3}{4 \pi} \frac{\left(x_{i}-y_{i}\right)\left(x_{j}-y_{j}\right)}{|x-y|^{2}} \cdot \frac{\left(x-y, n_{y}\right)}{|x-y|^{3}}, x \in \mathbb{R}^{3}, y \in \partial \mathcal{O},
$$

where $n_{y}$ is the outer normal vector at $y$. By the theory of integral equations with weak singularities (see, e.g., the references in [24]) we have that the function $\phi$ is uniquely defined by $\psi$ from (16) and the mapping $\psi \mapsto \phi$ is continuous from $\left[L_{2}(\Omega)\right]^{2}$ into $\left[L_{2}(\partial \mathcal{O})\right]^{3}$. One can also show (some details can be found in [24, Chapter 3, Section 2]) that

$$
\sup _{x \in \mathcal{O}} \int_{\partial \mathcal{O}}\left|K_{i j}(x, y)\right| d S_{y}+\sup _{y \in \partial \mathcal{O}} \int_{\mathcal{O}}\left|K_{i j}(x, y)\right| d x \leq C, \quad i, j=1,2,3 .
$$

Therefore we can apply the Schur test (see, e.g., Theorem 5.2 in [21]) to conclude that the mapping $\phi \mapsto v$ given by (15) is continuous from $\left[L_{2}(\partial \mathcal{O})\right]^{3}$ into $\left[L_{2}(\mathcal{O})\right]^{3}$. This implies (13).

We do not pretend that the boundary trace regularity stated in Proposition 2.1 are optimal. They are sufficient for our purposes and therefore we do not pursue the optimality issues. 


\subsection{Abstract representation of the problem}

We introduce the following spaces

$$
X=\left\{v \in\left[L_{2}(\mathcal{O})\right]^{3}: \operatorname{div} v=0 ; \gamma_{n} v \equiv(v, n)=0 \text { on } \partial \mathcal{O}\right\}
$$

and

$$
V=\left\{v \in\left[H^{1}(\mathcal{O})\right]^{3}: \operatorname{div} v=0 ; v=0 \text { on } S, \gamma_{n} v=0 \text { on } \Omega\right\} .
$$

We equip $X$ with $L_{2}$-type norm $\|\cdot\|_{\mathcal{O}}$ and denote by $(\cdot, \cdot)_{\mathcal{O}}$ the corresponding inner product. The space $V$ is endowed with the norm $\|\cdot\|_{V}=\|\nabla \cdot\|_{\mathcal{O}}$

Let $P_{L}$ be the Leray projector which maps the space $\left[L_{2}(\mathcal{O})\right]^{3}$ onto $X$. With this projector we rewrite (1) -(4) in $X$ as follows

$$
\partial_{t} v+A_{0}\left(v-N_{0} u_{t}\right)=G, \quad t>0,\left.\quad v\right|_{t=0}=v_{0},
$$

where $G=P_{L} \tilde{G} \in X, N_{0}$ is defined by (14), and $A_{0}=-\nu P_{L} \Delta$ is a positive self-adjoint operator with the domain

$$
\mathcal{D}\left(A_{0}\right)=\left\{v \in\left[H^{2}(\mathcal{O})\right]^{3}: \operatorname{div} v=0 ; v=0 \text { on } \partial \mathcal{O}\right\} .
$$

For the plate component we use the spaces

$$
Y=\left[L_{2}(\Omega)\right]^{2} \equiv L_{2}(\Omega) \times L_{2}(\Omega) \text { and } W=\left[H_{0}^{1}(\Omega)\right]^{2} .
$$

We denote by $\|\cdot\|_{\Omega}$ and $(\cdot, \cdot)_{\Omega}$ the norm and the inner product in $Y$.

In the space $Y$ for $\lambda>0$ we introduce the operator

$$
A=-\left[\begin{array}{cc}
(1+\lambda) \partial_{x_{1}}^{2}+\partial_{x_{2}}^{2} & \lambda \partial_{x_{1} x_{2}}^{2} \\
\lambda \partial_{x_{1} x_{2}}^{2} & \partial_{x_{1}}^{2}+(1+\lambda) \partial_{x_{2}}^{2}
\end{array}\right]
$$

with the domain $\mathcal{D}(A)=\left[\left(H^{2} \cap H_{0}^{1}\right)(\Omega)\right]^{2} \subset W$. One can see that $A$ is a positive operator in $Y$ generated by the form

$$
a(u, \hat{u})=\sum_{i=1}^{2} \int_{\Omega} \nabla u^{i} \nabla \hat{u}^{i} d \Omega+\lambda \int_{\Omega} \operatorname{div} u \cdot \operatorname{div} \hat{u} d \Omega \equiv\left(A^{1 / 2} u, A^{1 / 2} \hat{u}\right)_{\Omega},
$$

where $u=\left(u^{1} ; u^{2}\right)$ and $u=\left(\hat{u}^{1} ; \hat{u}^{2}\right)$ are from $W$. With this operator $A$ problem (77) and (8) can be written in the space $Y$ as follows,

$$
u_{t t}+A u+\gamma_{\Omega} v+f(u)=0, \quad t>0,\left.\quad u\right|_{t=0}=u_{0},\left.\quad u_{t}\right|_{t=0}=u_{1} .
$$

where $u=\left(u^{1} ; u^{2}\right)$, the operator $A$ is defined in (18),$\gamma_{\Omega}$ is given by (12), and $f(u)=\left(f^{1}(u) ; f^{2}(u)\right)$.

Now we consider the phase space $\mathcal{H}=X \times W \times Y$ with the inner product

$$
\left(U, U^{*}\right)_{\mathcal{H}}=\left(v, v^{*}\right)_{\mathcal{O}}+\left(A^{1 / 2} u_{0}, A^{1 / 2} u_{0}^{*}\right)_{\Omega}+\left(u_{1}, u_{1}^{*}\right)_{\Omega},
$$


where $U=\left(v ; u_{0} ; u_{1}\right)$ and $U^{*}=\left(v^{*} ; u_{0}^{*} ; u_{1}^{*}\right)$ are elements from $\mathcal{H}$.

We rewrite problem (17) and (19) as a first order equation for the phase variable $U=\left(v ; u ; u_{t}\right) \in \mathcal{H}$ of the form

$$
\frac{d U}{d t}+\mathcal{A} U+\mathcal{F}(U)=0, \quad t>0,\left.\quad U\right|_{t=0}=U_{0},
$$

where $\mathcal{F}(U)=(0 ; 0 ; f(u))$ and

$$
\mathcal{A}=\left[\begin{array}{ccc}
-\nu P_{L} \Delta & 0 & 0 \\
0 & 0 & -I \\
\gamma_{\Omega} & A & 0
\end{array}\right] \equiv\left[\begin{array}{ccc}
A_{0} & 0 & -A_{0} N_{0} \\
0 & 0 & -I \\
\gamma_{\Omega} & A & 0
\end{array}\right]
$$

with the domain

$$
\mathcal{D}(\mathcal{A})=\left\{\begin{array}{l|l}
U=\left[\begin{array}{c}
v \\
u_{0} \\
u_{1}
\end{array}\right] \in \mathcal{H} & \begin{array}{l}
A_{0}\left(v-N_{0} u_{1}\right) \equiv-\nu P_{L} \Delta v \in X \\
u_{1} \in W \\
A u_{0}+\gamma_{\Omega} v \in Y,\left.v\right|_{\Omega}=\left(u_{1} ; 0\right)
\end{array}
\end{array}\right\}
$$

To solve (20) we use methods from [29]. For this we first prove the following assertion.

Proposition 2.2 The operator $\mathcal{A}$ is a maximal accretive operator in $\mathcal{H}$. Moreover, $R(\mathcal{A})=\mathcal{H}$ and thus $\mathcal{A}$ is invertible and by the Lumer-Phillips theorem (see [29, $p$. 14]) generates $C_{0}$-semigroup of contractions in $\mathcal{H}$.

Proof. One can see that

$$
\left(\mathcal{A} U, U^{*}\right)_{\mathcal{H}}=\nu\left(\nabla v, \nabla v^{*}\right)_{\mathcal{O}}-\left(A u_{0}, u_{1}^{*}\right)_{\Omega}+\left(A u_{0}^{*}, u_{1}\right)_{\Omega},
$$

where $U=\left(v ; u_{0} ; u_{1}\right)$ and $U^{*}=\left(v^{*} ; u_{0}^{*} ; u_{1}^{*}\right)$ are elements from $\mathcal{D}(\mathcal{A})$. This implies that $(\mathcal{A} U, U)_{\mathcal{H}}=\nu\|\nabla v\|_{\mathcal{O}}^{2} \geq 0$ and thus $\mathcal{A}$ is accretive.

To prove maximality it suffices to show that $R(\mathcal{A})=\mathcal{H}$, i.e., to solve the equation of the form $\mathcal{A} U=F$ for $F \equiv\left(g ; h_{0} ; h_{1}\right) \in \mathcal{H}$. We obviously have that $U=\left(v ; u ;-h_{0}\right)$, where $v \in V$ solves

$$
\begin{aligned}
& -\nu \Delta v+\nabla p=g, \quad \operatorname{div} v=0 \quad \text { in } \mathcal{O} ; \\
& v=0 \text { on } S ; \quad v=\left(-h_{0} ; 0\right) \text { on } \Omega,
\end{aligned}
$$

with $h_{0} \in W=\left[H_{0}^{1}(\Omega)\right]^{2}$, and $u \in W$ satisfies the equation

$$
A u=-\gamma_{\Omega} v+h_{1}, \quad h_{1} \in Y=\left[L_{2}(\Omega)\right]^{2} .
$$

It follows from Proposition 2.1 that there exists $v \in\left[H^{3 / 2}(\mathcal{O})\right]^{3}$ satisfying (23) such that $\gamma_{\Omega} v \in\left[H^{-1 / 2}(\Omega)\right]^{2} \subset\left[\mathcal{D}\left(A^{1 / 4}\right)\right]^{\prime}$. Now we can solve equation (24) with respect to $u$. Thus $R(\mathcal{A})=\mathcal{H}$.

Remark 2.3 The argument above shows that

$$
\left.\mathcal{D}(\mathcal{A}) \subset\left\{\begin{array}{l|l}
U=\left[\begin{array}{c}
v \\
u_{0} \\
u_{1}
\end{array}\right.
\end{array}\right] \in \mathcal{H} \mid \begin{array}{l}
v \in\left[H^{3 / 2}(\mathcal{O})\right]^{3}, \gamma_{\Omega} v \in\left[H^{-1 / 2}(\Omega)\right]^{2}, \\
\left.v\right|_{S}=0,\left.v\right|_{\Omega}=\left(u_{1} ; 0\right), \\
u_{0} \in\left[\left(H^{3 / 2} \cap H_{0}^{1}\right)(\Omega)\right]^{2}, u_{1} \in\left[H_{0}^{1}(\Omega)\right]^{2}
\end{array}\right\} .
$$




\section{Well-posedness}

We assume that the plate force potential $\Phi(u)$ possesses the properties

- $\Phi(u) \in C^{2}\left(\mathbb{R}^{2}\right)$ is nonnegative polynomially bounded function, i.e.,

$$
\left|\frac{\partial \Phi(u)}{\partial u^{i} \partial u^{j}}\right| \leq C\left(1+|u|^{p}\right), \quad i, j=1,2, \quad u=\left(u^{1}, u^{2}\right) \in \mathbb{R}^{2},
$$

for some $C, p \geq 0$.

- The following dissipativity condition holds: for any $\delta>0$ there exists $c_{1}(\delta)>0$ and $c_{2}(\delta) \geq 0$ such that

$$
\sum_{i=1,2} u^{i} f^{i}(u)-c_{1}(\delta) \Phi(u)+\delta|u|^{2} \geq-c_{2}(\delta) \text { with } f^{i}(u)=\frac{\partial \Phi(u)}{\partial u^{i}}
$$

As examples of a such potential $\Phi(u)$ we can consider

$$
\Phi(u)=\psi_{0}\left(\left|u^{1}\right|^{2}+\left|u^{2}\right|^{2}\right) \text { or } \Phi(u)=\psi_{1}\left(u^{1}\right)+\psi_{2}\left(u^{2}\right),
$$

where $\psi_{i}(s)$ are nonnegative functions from $C^{2}(\mathbb{R})$ such that

(a) $\left|\psi^{\prime \prime}(s)\right| \leq C\left(1+|u|^{q}\right)$ for some $C, q \geq 0$;

(b) $s \psi_{i}^{\prime}(s)-c_{0} \psi_{i}(s) \geq-c_{1}$ for some $c_{0}>0$ and $c_{1} \geq 0$.

For instance, $\psi_{i}(s)$ can be polynomials of even degree with a positive leading coefficient and and with sufficiently large free term.

We also note that under the assumption in (25) the nonlinear force

$$
f(u)=\left(f^{1}(u) ; f^{2}(u)\right)=\left(\frac{\partial \Phi(u)}{\partial u^{1}} ; \frac{\partial \Phi(u)}{\partial u^{2}}\right)
$$

in (19) satisfies the following local Lipschits property

$$
\|f(u)-f(\hat{u})\|_{Y} \leq C_{\sigma}\left(1+\|u\|_{1-\sigma / p, \Omega}^{p}+\|\hat{u}\|_{1-\sigma / p, \Omega}^{p}\right)\|u-\hat{u}\|_{\sigma, \Omega},
$$

for every $0<\sigma<1$ and $u, \hat{u} \in W$, where $\|\cdot\|_{\sigma, \Omega}$ denotes the norm in the space $\left[H^{\sigma}(\Omega)\right]^{2}$. To see this we note that (25) implies the estimate

$$
\left|f^{i}(u)-f^{i}(\hat{u})\right| \leq C\left(1+|u|^{p}+|\hat{u}|^{p}\right)|u-\hat{u}|, \quad u, \hat{u} \in \mathbb{R}^{2} .
$$

Therefore the Hölder inequality and the embedding $H^{\sigma}(\Omega) \subset L_{2 /(1-\sigma)}(\Omega)$ for $0<\sigma<1$ imply (28).

Following the standard semigroup approach (see, e.g., [29] or [31]) we give the following definition. 
Definition 3.1 The function $U(t)=\left(v(t) ; u(t) ; u_{t}(t)\right) \in C(0, T ; \mathcal{H})$ such that $U(0)=U_{0}=\left(v ; u_{0} ; u_{1}\right)$ is said to be

- strong solution to problem (20) on an interval $[0, T]$ if (i) $U(t) \in \mathcal{D}(\mathcal{A})$ for almost all $t \in[0, T]$, (ii) $U_{t} \in L_{1}(0, T ; \mathcal{H}$ ), and (iii) (20) is satisfied as an equality in $\mathcal{H}$ for almost all $t \in[0, T]$;

- generalized solution to problem (20) if there exist a sequence of initial data $U_{0}^{n}$ and the corresponding strong solutions $U^{n}(t)$ such that

$$
\lim _{n \rightarrow 0} \max _{t \in[0, T]}\left\|U^{n}(t)-U(t)\right\|_{\mathcal{H}}=0 .
$$

Theorem 3.2 Let $U_{0} \in \mathcal{H}$. Then for any interval $[0, T]$ there exists a unique generalized solution $U(t)=\left(v(t) ; u(t) ; u_{t}(t)\right)$ such that $v \in L_{2}(0, T ; V)$ and the energy balance equality

$$
\mathcal{E}\left(v(t), u(t), u_{t}(t)\right)+\nu \int_{0}^{t}\|\nabla v\|_{\mathcal{O}}^{2} d \tau=\mathcal{E}\left(v_{0}, u_{0}, u_{1}\right)+\int_{0}^{t}(G, v)_{\mathcal{O}} d \tau
$$

holds for $t>0$, where

$$
\mathcal{E}\left(v(t), u(t), u_{t}(t)\right)=\frac{1}{2}\|v(t)\|_{\mathcal{O}}^{2}+E\left(u(t), u_{t}(t)\right)
$$

with the energy $E\left(u, u_{t}\right)$ of the plate given by

$$
E\left(u, u_{t}\right)=\frac{1}{2}\left(\left\|u_{t}\right\|_{\Omega}^{2}+\left\|A^{1 / 2} u\right\|_{\Omega}^{2}\right)+\int_{\Omega} \Phi(u(x)) d \Omega .
$$

Moreover,

- Any generalized generalized solution is also mild, i.e.

$$
U(t)=e^{-t \mathcal{A}} U_{0}+\int_{0}^{t} e^{-(t-\tau) \mathcal{A}} \mathcal{F}(U(\tau)) d \tau, \quad t>0
$$

- There exists a constant $a_{R, T}>0$ such that for any couple of generalized solutions $U(t)=\left(v(t) ; u(t) ; u_{t}(t)\right)$ and $\hat{U}(t)=\left(\hat{v}(t) ; \hat{u}(t) ; \hat{u}_{t}(t)\right)$ with the initial data possessing the property $\left\|U_{0}\right\|_{\mathcal{H}},\left\|\hat{U}_{0}\right\|_{\mathcal{H}} \leq R$ we have

$$
\|U(t)-\hat{U}(t)\|_{\mathcal{H}}^{2}+\int_{0}^{t}\|\nabla(v-\hat{v})\|_{\mathcal{O}}^{2} d \tau \leq a_{R, T}\left\|U_{0}-\hat{U}_{0}\right\|_{\mathcal{H}}^{2}
$$

for every $t \in[0, T]$.

- The solution $U(t)$ is strong if $U_{0} \in \mathcal{D}(\mathcal{A})$. 
Proof. By Proposition 2.2 the linear part of (20) generates a strongly continuous semigroup. By (28) we also have that the nonlinear part in (201) is locally Lipschitz on $\mathcal{H}$. Therefore the existence of (local) strong and generalized solutions follows from Theorem 6.1.6 [29] and from the argument provided in the proof of Theorem 6.1.4 [29]. The latter theorem also means that generalized solutions are mild on the existence interval.

Now we consider strong solutions on the existence interval and establish the energy relation. A simple calculation gives

$$
\frac{1}{2} \frac{d}{d t}\|v(t)\|_{\mathcal{O}}^{2}+\nu\|\nabla v(t)\|_{\mathcal{O}}^{2}=\nu \int_{\Omega}\left[\frac{\partial v^{1}}{\partial n} u_{t}^{1}+\frac{\partial v^{1}}{\partial n} u_{t}^{2}\right] d \Omega+\int_{\mathcal{O}} v G d x .
$$

It is also clear that for plate we have that

$$
\frac{d}{d t} E\left(u(t), u_{t}(t)\right)+\nu \int_{\Omega}\left[\frac{\partial v^{1}}{\partial x_{3}} u_{t}^{1}+\frac{\partial v^{1}}{\partial x_{3}} u_{t}^{2}\right] d \Omega=0
$$

The sum of equalities (33) and (34) along with the relation $n=(0 ; 0 ; 1)$ on $\Omega$ after integration gives the energy equality in (29) for strong solutions on the existence interval.

It follows from (29) and (25) that

$$
\|U(t)\|_{\mathcal{H}}^{2} \leq C_{R}+\int_{0}^{t}\|G(\tau)\|_{\mathcal{O}}^{2} d \tau, \quad\left\|U_{0}\right\|_{\mathcal{H}} \leq R
$$

on the existence interval. This implies (see, e.g., Theorem 6.1.4 [29]) that both strong and generalized solutions cannot blow up and therefore they can be extended on $\mathbb{R}_{+}$.

By the same argument as in the proof of the energy equality using relations (28) and (35) we can prove that

$$
\begin{aligned}
& \|U(t)-\hat{U}(t)\|_{\mathcal{H}}^{2}+2 \nu \int_{0}^{t}\|\nabla(v-\hat{v})\|_{\mathcal{O}}^{2} d \tau \\
\leq & \left\|U_{0}-\hat{U}_{0}\right\|_{\mathcal{H}}^{2}+c_{R, T} \int_{0}^{t}\|U(t)-\hat{U}(t)\|_{\mathcal{H}}\left\|u_{t}-\hat{u}_{t}\right\|_{\Omega} d \tau, \quad t \in[0, T] .
\end{aligned}
$$

Using the standard trace theorem from the boundary condition in (4) we have that $\left\|u_{t}-\hat{u}_{t}\right\|_{\Omega} \leq C\|\nabla(v-\hat{v})\|_{\mathcal{O}}$. Therefore applying the Gronwall type argument we obtain (32).

The relation in (32) allows us to make limit transition in energy relation (29) from strong to generalized solutions and to conclude the proof of Theorem 3.2 ,

Theorem 3.2 makes it possible to define a dynamical system $\left(\mathcal{H}, S_{t}\right)$ with the phase space $\mathcal{H}=X \times W \times Y$ and the evolution operator $S_{t}$ defined by

$$
S_{t} U_{0}=U(t)=\left(v(t) ; u(t) ; u_{t}(t)\right),
$$

where $U(t)$ is a generalized solution to (20) with initial data $U_{0} \in \mathcal{H}$. 


\section{Main result}

Our main result is the following theorem.

Theorem 4.1 The dynamical system $\left(\mathcal{H}, S_{t}\right)$ generated by (20) possesses a compact global attractor $\mathfrak{A}$ of finite fractal dimension. Moreover, for any trajectory $\left\{U(t)=\left(v(t) ; u(t) ; u_{t}(t)\right): t \in \mathbb{R}\right\}$ from the attractor $\mathfrak{A}$ we have that $U \in C(\mathbb{R} ; \mathcal{D}(\mathcal{A})) \cap C^{1}(\mathbb{R} ; \mathcal{H})$ and

$$
\begin{aligned}
\sup _{t \in \mathbb{R}}\left\{\left\|v_{t}(t)\right\|_{\mathcal{O}}+\left\|P_{L} \Delta v(t)\right\|_{\mathcal{O}}\right. \\
\left.\quad+\left\|A^{1 / 2} u_{t}(t)\right\|_{\Omega}+\left\|u_{t t}(t)\right\|_{\Omega}+\left\|A u(t)+\gamma_{\Omega} v(t)\right\|_{\Omega}\right\} \leq C_{\mathfrak{A}} .
\end{aligned}
$$

We recall (see, e.g., [4, 9, 32]) that a global attractor of a dynamical system $\left(\mathcal{H}, S_{t}\right)$ is a bounded closed set $\mathfrak{A} \subset \mathcal{H}$ which is invariant (i.e., $S_{t} \mathfrak{A}=\mathfrak{A}$ ) and uniformly attracts all other bounded sets:

$$
\lim _{t \rightarrow \infty} \sup \left\{\operatorname{dist}\left(S_{t} U, \mathfrak{A}\right): U \in B\right\}=0 \quad \text { for any bounded set } B \text { in } \mathcal{H} .
$$

The fractal dimension $\operatorname{dim}_{f}^{\mathcal{H}} \mathfrak{A}$ of a set $\mathfrak{A}$ in the space $\mathcal{H}$ is defined as

$$
\operatorname{dim}_{f}^{\mathcal{H}} \mathcal{A}=\limsup _{\varepsilon \rightarrow 0} \frac{\ln N(\mathfrak{A}, \varepsilon)}{\ln (1 / \varepsilon)}
$$

where $N(\mathfrak{A}, \varepsilon)$ is the minimal number of closed sets in $\mathcal{H}$ of diameter $2 \varepsilon$ needed to cover the set $\mathfrak{A}$.

To prove the existence of a compact global attractor for $\left(\mathcal{H}, S_{t}\right)$ it is sufficient (see, e.g., [20, 32]) to show that the system $\left(\mathcal{H}, S_{t}\right)$ is dissipative and asymptotically smooth. We recall (see [4, 9, 32]) that the system is dissipative if there exists a bounded absorbing set $\mathcal{B}_{0}$ in $\mathcal{H}$. A set $\mathcal{B}_{0}$ is said to be absorbing for $\left(\mathcal{H}, S_{t}\right)$ if for any bounded set $\mathcal{B} \subset \mathcal{H}$ there exists time $t_{\mathcal{B}}$ such that $S_{t} \mathcal{B} \subset \mathcal{B}_{0}$ for all $t \geq t_{\mathcal{B}}$. A system $\left(\mathcal{H}, S_{t}\right)$ is said to be asymptotically smooth if for any closed bounded forward invariant set $B \subset \mathcal{H}$ there exists a compact set $\mathcal{K}=\mathcal{K}(B)$ which uniformly attracts $B$ :

$$
\lim _{t \rightarrow \infty} \sup \left\{\operatorname{dist}\left(S_{t} U, \mathcal{K}\right): U \in B\right\}=0 .
$$

Below to prove dissipativity we use an appropriate Lyapunov function. As fo asymptotic smoothness of the system and finite-dimensionality of the attractor, we rely on recently developed approach based on stabilizability estimates (see [12, 14] and the references therein).

\subsection{Dissipativity}

Proposition 4.2 The system $\left(\mathcal{H}, S_{t}\right)$ is dissipative. Moreover, there exists a bounded forward invariant absorbing set. 
Proof. Let $S_{t} U_{0}=U(t)=\left(v(t) ; u(t) ; u_{t}(t)\right)$. We consider the following Lyapunov type function

$$
\mathcal{W}\left(v, u, u_{t}\right)=\mathcal{E}\left(v, u, u_{t}\right)+\eta\left[\left(u, u_{t}\right)_{\Omega}+\left(v, N_{0} u\right)_{\mathcal{O}}\right],
$$

where the energy $\mathcal{E}$ is defined by (30) and the operator $N_{0}$ id given by (14). The parameter $\eta$ will be chosen later.

In the further calculations we deal with strong solutions.

The trace theorem and the boundary condition on $\Omega$ given in (4) imply that $\left\|u_{t}\right\|_{\Omega}^{2} \leq C\|\nabla v\|_{\mathcal{O}}^{2}$ and therefore it follows from energy relation (29) that

$$
\frac{d}{d t} \mathcal{E}\left(v(t), u(t), u_{t}(t)\right)+c_{0}\left(\left\|u_{t}\right\|_{\Omega}^{2}+\|\nabla v\|_{\mathcal{O}}^{2}\right) \leq c_{1}\|G\|_{\mathcal{O}}^{2}
$$

with some positive constants $c_{i}$. Using (17) and (19) we also have that

$$
\begin{aligned}
& \frac{d}{d t}\left[\left(u, u_{t}\right)_{\Omega}+\left(v, N_{0} u\right)_{\mathcal{O}}\right] \\
= & \left\|u_{t}\right\|_{\Omega}^{2}+\left(u, u_{t t}\right)_{\Omega}+\left(v_{t}, N_{0} u\right)_{\mathcal{O}}+\left(v, N_{0} u_{t}\right)_{\mathcal{O}} \\
= & \left\|u_{t}\right\|_{\Omega}^{2}-\left\|A^{1 / 2} u\right\|_{\Omega}^{2}-(f(u), u)_{\Omega}-\left(\gamma_{\Omega} v, u\right)_{\Omega} \\
& +\left(\nu \Delta v+G, N_{0} u\right)_{\mathcal{O}}+\left(v, N_{0} u_{t}\right)_{\mathcal{O}} \\
= & \left\|u_{t}\right\|_{\Omega}^{2}-\left\|A^{1 / 2} u\right\|_{\Omega}^{2}-(f(u), u)_{\Omega} \\
& -\nu\left(\nabla v, \nabla N_{0} u\right)_{\mathcal{O}}+\left(G, N_{0} u\right)_{\mathcal{O}}+\left(v, N_{0} u_{t}\right)_{\mathcal{O}} .
\end{aligned}
$$

Here above we also use the fact that $A_{0}\left(v-N_{0} u_{t}\right)=-P_{L} \Delta v$ and the Green formula:

$$
\nu\left(\Delta v, N_{0} u\right)_{\mathcal{O}}=-\nu\left(\nabla v, \nabla N_{0} u\right)_{\mathcal{O}}+\left(\gamma_{\Omega} v, u\right)_{\Omega} .
$$

Therefore using Proposition 2.1 we obtain that

$$
\begin{aligned}
& \frac{d}{d t}\left[\left(u, u_{t}\right)_{\Omega}+\left(v, N_{0} u\right)_{\mathcal{O}}\right] \\
\leq & 2\left\|u_{t}\right\|_{\Omega}^{2}-(1-\delta)\left\|A^{1 / 2} u\right\|_{\Omega}^{2}-(f(u), u)_{\Omega}+c_{\delta}\left(\|\nabla v\|_{\mathcal{O}}^{2}+\|G\|_{\mathcal{O}}^{2}\right)
\end{aligned}
$$

for every $\delta>0$. By our hypotheses (see (26) ) there is $c_{f}^{*}>0$ and $c_{f} \geq 0$ such that

$$
(f(u), u)_{\Omega}-c_{f}^{*} \int_{\Omega} \Phi(u) d \Omega+\frac{1}{2}\left\|A^{1 / 2} u\right\|_{\Omega}^{2} \geq-c_{f} .
$$

Therefore from (38) we have that

$$
\begin{aligned}
& \frac{d}{d t}\left[\left(u, u_{t}\right)_{\Omega}+\left(v, N_{0} u\right)_{\mathcal{O}}\right] \\
\leq & 2\left\|u_{t}\right\|_{\Omega}^{2}-\frac{1}{4}\left\|A^{1 / 2} u\right\|_{\Omega}^{2}-c_{f}^{*} \int_{\Omega} \Phi(u) d \Omega+c_{1 / 4}\left(\|\nabla v\|_{\mathcal{O}}^{2}+\|G\|_{\mathcal{O}}^{2}\right)+c_{f} .
\end{aligned}
$$


After selecting $\eta>0$ small enough, this relation along with (37) implies that

$$
\frac{d}{d t} \mathcal{W}\left(v(t), u(t), u_{t}(t)\right)+c_{0} \mathcal{W}\left(v(t), u(t), u_{t}(t)\right) \leq c_{1}\left(c_{f}+\|G\|_{\mathcal{O}}^{2}\right)
$$

with some $c_{0}, c_{1}>0$. This yields

$$
\mathcal{W}\left(v(t), u(t), u_{t}(t)\right) \leq \mathcal{W}\left(v_{0}, u_{0}, u_{1}\right) e^{-c_{0} t}+\frac{c_{1}}{c_{0}}\left(c_{f}+\|G\|_{\mathcal{O}}^{2}\right)\left[1-e^{-c_{0} t}\right]
$$

For $\eta>0$ small enough we definitely have that

$$
\frac{1}{2} \mathcal{E}\left(v, u, u_{t}\right) \leq \mathcal{W}\left(v, u, u_{t}\right) \leq 2 \mathcal{E}\left(v, u, u_{t}\right)
$$

Therefore the standard argument (see, e.g., [4, 9, 32]) yields dissipativity with a forward invariant bounded absorbing set.

The argument given above allows us to obtain the following assertion on asymptotic stability.

Proposition 4.3 If $G \equiv 0$ and relation (26) holds with $c_{2}(\delta) \equiv 0$, Then there exist $c_{R}>0$ and $\alpha>0$ such that

$$
\left\|S_{t} U\right\|_{\mathcal{H}} \leq c_{R} e^{-\alpha t} \text { for any } U \in \mathcal{H} \text { such that }\|U\|_{\mathcal{H}} \leq R .
$$

This means that in this case the global attractor $\mathfrak{A}$ consists of a single point, $\mathfrak{A}=\{(0 ; 0 ; 0)\}$, which is exponentially attractive.

Proof. In the case considered we have $c_{f}=0$ in (39) and also $G \equiv 0$. Thus (41) has the form

$$
\mathcal{W}\left(v(t), u(t), u_{t}(t)\right) \leq \mathcal{W}\left(v_{0}, u_{0}, u_{1}\right) e^{-c_{0} t}, \quad t>0 .
$$

Hence (42) implies (43).

We note that the hypotheses of Proposition 4.3 holds true if $G \equiv 0$ and relation (27) is valid with $\psi_{i} \in C^{2}(\mathbb{R})$ possessing the additional properties: $\psi_{i}(s) \geq \psi_{i}(0)=0$ and $\psi_{i}^{\prime}(s)$ is non-decreasing function.

As a consequence of Proposition 4.3 for $f(u) \equiv 0$ we have the following assertion, which enforces Proposition 2.2.

Corollary 4.4 The operator $\mathcal{A}$ given by (21) and (22) generates an exponentially stable $C_{0}$-semigroup of contractions $e^{-t \mathcal{A}}$ in $\mathcal{H}$. In particular, there exists positive $C, \alpha$ such that

$$
\left\|e^{-t \mathcal{A}} U\right\|_{L(\mathcal{H}, \mathcal{H})} \leq C e^{-\alpha t} \text { for all } t>0 .
$$

This corollary improves the result in [19] which states the strong stability only. 


\subsection{Quasi-stability}

We use the method developed in [10] (see also [12, 14] and the references therein) to obtain asymptotic smoothness and finiteness of fractal dimension of the attractor. This method based on quasi-stability properties of the system and involves the so-called stabilizability estimate.

\section{Proposition 4.5 (Stabilizability estimate) Let}

$$
S_{t} U_{0} \equiv U(t)=\left(v(t) ; u(t) ; u_{t}(t)\right) \text { and } S_{t} U_{0}^{*} \equiv U^{*}(t)=\left(v^{*}(t) ; u^{*}(t) ; u_{t}^{*}(t)\right)
$$

be two semi-trajectories such $\left\|S_{t} U_{0}\right\|_{\mathcal{H}},\left\|S_{t} U_{0}^{*}\right\|_{\mathcal{H}} \leq R$ for all $t>0$ and for some $R>0$. Then there exist positive constants $c_{0}, \omega$ and $c_{R}$ such that

$$
\left\|S_{t} U_{0}-S_{t} U_{0}^{*}\right\|_{\mathcal{H}} \leq c_{0} e^{-\omega t}\left\|U_{0}-U_{0}^{*}\right\|_{\mathcal{H}}+c_{R} \int_{0}^{t} e^{-\omega(t-\tau)}\left\|u(\tau)-u^{*}(\tau)\right\|_{\Omega} d \tau
$$

for all $t>0$.

Proof. Using the representation (31), the exponential stability of the linear semigroup $e^{-t \mathcal{A}}$ given by (44), and also the local Lipschitz property in (28) we obtain that

$$
\left\|S_{t} U_{0}-S_{t} U_{0}^{*}\right\|_{\mathcal{H}} \leq C e^{-\alpha t}\left\|U_{0}-U_{0}^{*}\right\|_{\mathcal{H}}+C_{R} \int_{0}^{t} e^{-\alpha(t-\tau)}\|z(\tau)\|_{\sigma, \Omega} d \tau
$$

for all $t>0$, where $z(t)=u(t)-u^{*}(t)$ and $0<\sigma<1$, By interpolation,

$$
\|z\|_{\sigma, \Omega} \leq \delta\left\|A^{1 / 2} z\right\|_{\Omega}+C_{\delta}\|z\|_{\Omega} \leq \delta\left\|S_{t} U_{0}-S_{t} U_{0}^{*}\right\|_{\mathcal{H}}+C_{\delta}\|z\|_{\Omega}
$$

for every $\delta>0$. Substituting this relation in (46) and applying the Gronwall type argument with an appropriate choice $\delta$ we obtain (45).

We note that the property stated in Proposition 4.5 means that the system $\left(\mathcal{H}, S_{t}\right)$ is quasi-stable (in the sense of [14, Definition 7.9.2]). This observation make it possible to obtain several important dynamical properties at the abstract level.

\subsection{Completion of Theorem 4.1}

Using the stabilizability estimate in (45) via the Ceron-Lopes type criteria (see [12, Corollary 2.7]) we can easily prove that $\left(\mathcal{H}, S_{t}\right)$ is asymptotically smooth (for some details for similar systems see, e.g., [12, 13] and also [14, Section 7.9] at the abstract level). Thus there exists a compact global attractor $\mathfrak{A}$ for $\left(\mathcal{H}, S_{t}\right)$.

This attractor has a finite fractal dimension. To see this one should apply the same argument as in [12, Theorem 4.3], see also the argument 
given in the proof of Theorem 4.1 in [13] for the case of thermoelastic plate models.

The regularity property in (36) follows by the same argument as in the proof of [12, Theorem 4.17], see also the proof of Theorem 6.2 [13] and argument given in [14, Section 7.9.2] for the abstract quasi-stable systems.

Thus the proof of Theorem 4.1 is complete.

Remark 4.6 As in [12] and [14, Section 7.9] we can use the stablizability estimate to construct exponential fractal attractor (whose dimension is finite in some extended space) and prove the existence of finite number of determining functionals supported by displacement component of the plate. We also note that using the same approach as in [12, Theorem 4.23] (see also [14, Section 8.7]) and assuming additional smoothness of the potential

$\Phi$ we can obtain higher regularity of time derivatives of the trajectories from the attractor.

\section{References}

[1] G. Avalos, The strong stability and instability of a fluid-structure semigroup, Appl. Math. Optim., 55 (2007), 163-184.

[2] G. Avalos, R. Triggiani, The coupled PDE system arising in fluidstructure interaction. I. Explicit semigroup generator and its spectral properties, in: Fluids and Waves, Contemp. Math., vol. 440, AMS, Providence, RI, 2007, 15-54.

[3] G. Avalos and R. Triggiani, Semigroup well-posedness in the energy space of a parabolic hyperbolic coupled Stokes-Lamé PDE system of fluid-structure interaction, Discr. Contin. Dyn. Sys., Ser.S, 2 (2009), 417-447.

[4] A.V. Babin, M.I. Vishik. Attractors of Evolution Equations. NorthHolland, Amsterdam, 1992.

[5] V. Barbu, Z. Grujić, I. Lasiecka, A. Tuffaha, Existence of the energylevel weak solutions for a nonlinear fluid-structure interaction model, in: Fluids and Waves, Contemp. Math., vol. 440, AMS, Providence, RI, 2007, 55-82.

[6] V. Barbu, Z. Grujić, I. Lasiecka, A. Tuffaha, Smoothness of weak solutions to a nonlinear fluid-structure interaction model, Indiana Univ. Math. J. 57 (2008), 1173-207.

[7] F. Bucci, I. Chueshov, Long-time dynamics of a coupled system of nonlinear wave and thermoelastic plate equations, Discr. Cont. Dyn.Systems 22 (2008), 557-586. 
[8] A. Chambolle, B. Desjardins, M. Esteban, C. Grandmont, Existence of weak solutions for the unsteady interaction of a viscous fluid with an elastic plate. J. Math. Fluid Mech. 7 (2005), 368-404.

[9] I. Chueshov, Introduction to the Theory of Infinite-Dimensional Dissipative Systems. Acta, Kharkov, 1999 (in Russian); English translation: Acta, Kharkov, 2002; http://www.emis.de/monographs/Chueshov/.

[10] I. Chueshov and I. Lasiecka, Attractors for second order evolution equations, J. Dynam. Diff. Eqs., 16 (2004), 469-512.

[11] I. Chueshov and I. Lasiecka, Global attractors for Mindlin-Timoshenko plates and for their Kirchhoff limits, Milan J. Math., 74 (2006), 117138.

[12] I. Chueshov and I. Lasiecka, Long-Time Behavior of Second Order Evolution Equations with Nonlinear Damping, Memoirs of AMS, vol.195, no. 912, AMS, Providence, RI, 2008.

[13] I. Chueshov and I. Lasiecka, Long-time behaviour of von Karman thermoelastic plates, Applied Math. Optim., 58 (2008), 195-241.

[14] I. Chueshov and I. Lasiecka, Von Karman Evolution Equations, Springer, New York, 2010; in print.

[15] D. Coutand, S. Shkoller, Motion of an elastic solid inside an incompressible viscous fluid, Arch. Ration. Mech. Anal. 176 (2005) 25-102.

[16] Q. Du, M.D. Gunzburger, L.S. Hou, J. Lee, Analysis of a linear fluidstructure interaction problem, Discrete Contin. Dyn. Syst. 9 (2003), $633-650$.

[17] M. Grobbelaar-Van Dalsen, On a fluid-structure model in which the dynamics of the structure involves the shear stress due to the fluid, $J$. Math. Fluid Mech. 10 (2008), 388-401.

[18] M. Grobbelaar-Van Dalsen, A new approach to the stabilization of a fluid-structure interaction model, Applicable Analysis 88 (2009), 10531065 .

[19] M. Grobbelaar-Van Dalsen, Strong stability for a fluidstructure model, Math. Methods Appl. Sci. 32 (2009), 1452-1466.

[20] J.K. Hale, Asymptotic Behavior of Dissipative Systems. Amer. Math. Soc., Providence, RI, 1988.

[21] P.R. Halmos, V.S Sunder, Bounded Integral Operators on $L^{2}$ Spaces, Springer, Berlin, 1978. 
[22] B. Kellogg, Properties of solutions of elliptic boundary value problems, in: The Mathematical Foundations of the Finite Element Method with Applications to Partial Differential Equations, A.K. Aziz (Ed.), Academic Press, New York, 1972, pp. 47-81.

[23] N. Kopachevskii, Yu. Pashkova, Small oscillations of a viscous fluid in a vessel bounded by an elastic membrane, Russian J. Math. Phys. 5 (1998), no.4, 459-472.

[24] O. Ladyzhenskaya, Mathematical Theory of Viscous Incompressible Flow, GIFML, Moscow, 1961 (1st Russian edition); Nauka, Moscow, 1970 (2nd Russian edition); Gordon and Breach, New York, 1963 and 1969 (English translations of the 1st Russian edition).

[25] J. Lagnese, Boundary Stabilization of Thin Plates, SIAM, Philadelphia, 1989.

[26] J. Lagnese, Modeling and stabilization of nonlinear plates, Int. Ser. Num. Math., 100 (1991), 247-264.

[27] J. Lagnese and J.L.Lions, Modeling, Analysis and Control of Thin Plates, Masson, Paris, 1988.

[28] I. Lasiecka and R. Triggiani, Control Theory for Partial Differential Equations, Cambridge University Press, Cambridge, 2000.

[29] A. Pazy, Semigroups of Linear Operators and Applications to Partial Differential Equations, Springer, New York, 1986.

[30] T.J.Pedley, The Fluid Mechanics of Large Blood Vessels, Cambridge University Press, Cambridge, 1980.

[31] R. Showalter, Monotone Operators in Banach Spaces and Nonlinear Partial Differential Equations, AMS, Providence, 1997.

[32] R. Temam, Infinite-Dimensional Dynamical Dystems in Mechanics and Physics, Springer, New York, 1988.

[33] R. Temam, Navier-Stokes Equations: Theory and Numerical Analysis, Reprint of the 1984 edition, AMS Chelsea Publishing, Providence, RI, 2001. 